\title{
SUPPRESSING UNDESIRED VIBRATION OF 3R ROBOT ARMS USING IMPACT DAMPERS
}

\author{
Mohammad Amin Rashidifar ${ }^{1}$ And Darvish Ahmadi ${ }^{2}$ \\ ${ }^{1}$ Department of Mechanical Engineering, Islamic Azad University, SHADEGAN Branch, \\ FATHOLMOBIN BLV, SHADEGAN, Khuzestan 6431863758, Iran \\ Department of Mechanical Engineering, Islamic Azad University, SHADEGAN Branch, \\ FATHOLMOBIN BLV, SHADEGAN, Khuzestan 6431863758, Iran
}

\begin{abstract}
The behavior of a vibration system suppressed with an impact damper is investigated, where the impact damper is simplified as a combination of spring and viscous damping. The analytical answer for the contact duration is developed using iteration perturbation technique. Iteration perturbation is a new analytical technique which can solve strongly nonlinear equations. In the presented work an impact damper is used to reduce the undesired vibration of an industrial robot arm. For this reason a $3 R$ robot is equipped with a simple model of a single unit impact damper which is constructed using spring, mass and viscous damper. The output forces of robot with and without impact damper are compared and effects of using the impact damper on correct application of the robot are shown.
\end{abstract}

\section{Keywords}

Impact damper, Iteration perturbation, $3 R$ robot

\section{INTRODUCTION}

An impact damper is a lightweight auxiliary device which consists of a container with a loose mass. In impact dampers, the secondary mass (loose mass) absorbs kinetic energy of the main vibratory system and converts it into heat through inelastic collisions between the secondary mass and the enclosure. This damper is effective for discrete and continuous systems in resonant operation and produces maximum displacement reduction during two impacts per cycle of motion. Applications of the impact dampers to reduce excessive vibration of industrial machinery have been extensively investigated by researchers [1]. The reason for this interest lies in the following features of an impact damper:

- Small and simple in construction.

- Easy to mount on the main vibratory systems.

- No need to adjust parameters of an impact damper to the vibratory characteristics of the main vibratory systems.

- Effective application to suppress excess vibration.

In the past few years, behavior of impact dampers has been investigated experimentally, analytically and numerically [6-11]. 
The effects of using impact dampers on better control of mechanical systems are investigated for many years [2]. For example, effectiveness of these systems on suppressing undesired vibrations of a cantilever beam was considered by many researchers [3]. But application of these systems on more complicated systems for example robot arms was not explored. Son et al. [12] proposed active momentum exchange impact dampers to suppress the first large peak value of the acceleration response due to a shock load.

In this paper the governing equation of motion of a vibratory system which is equipped with an impact damper is solved using a combination of nonlinear normal modes method and iteration perturbation $[4,5]$. Iteration perturbation is a new perturbation technique coupling with the iteration method. It can solve strongly nonlinear equations, accurately. Contact duration can be achieved by employing the discussed method and is used for finding motion of primary mass contacting the barriers. Therefore, the contact time is taken into consideration. This feature is important for a resilient rather than a rigid impact damper when the noise issue is concerned. Finally, the attenuating the undesired vibration of the tip of a robot arm is investigated. For this reason, a 3R robot is modeled in SIMMECHANICS toolbox of MATLAB. The robot is equipped with a simple model of a single unit impact damper which is constructed using spring, mass and viscous damper. The output forces of robot with and without impact damper are compared.

\section{MATHEMATICAL MODELING}

A simple model of a vibratory system consist of an oscillator with linear stiffness $\mathrm{K}$, mass $\mathrm{M}$, viscous damping $\mathrm{C}$ and an impact damper with mass $\mathrm{m}$ and clearance $\mathrm{d}$ is considered. $\mathrm{A}$ schematic model of this system is shown in figure 1 .

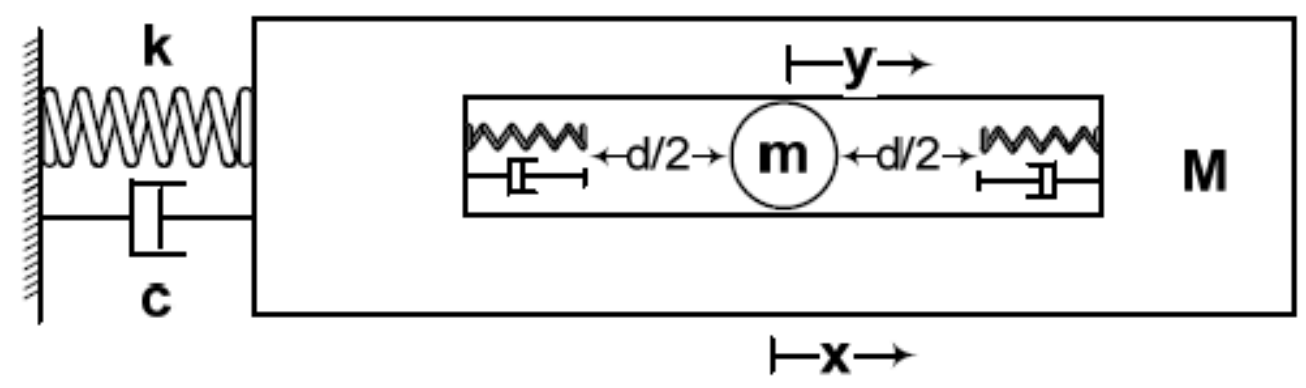

Figure1. Schematic of an impact damper

Motion of the vibratory system can be divided to two separate motions:

- Motion of impact damper between barriers.

- Motion of impact damper when it collides with barriers.

The differential equations of motion between impacts are shown as:

$M \ddot{x}+c \dot{x}+k x=0$

$$
m \ddot{y}=0
$$


So, the motion of the primary mass between impacts $i$ and $i+1$ can be written as follows:

$x\left(t^{\prime}\right)=e^{-\xi \omega t}\left[\left[\frac{\dot{x}_{i a}}{\sqrt{1-\zeta^{2}} \omega}+\frac{\xi}{\sqrt{1-\zeta^{2}}} x_{i}\right] \sin \left(\sqrt{1-\zeta^{2}} \omega t^{\prime}\right)+x_{i} \cos \left(\sqrt{1-\zeta^{2}} \omega t^{\prime}\right)\right.$

Where

$\omega^{2}=\sqrt{\frac{k}{m}}, \xi=\frac{c}{2 \sqrt{k m}}$ and $t^{\prime}=t-t_{i}$

And motion of the impact mass can be formulated as follows:

$y=\dot{y}_{i a} t^{\prime}+y_{i a}$

The subscript $\mathrm{i}, \mathrm{i}_{\mathrm{a}}, \mathrm{i}_{\mathrm{b}}$ represent the value of variables at contact, just after and just before the $\mathrm{i}^{\text {th }}$ contact, respectively. Impact occurs only when

$|x-y|=\frac{d}{2}$

Velocities after $i^{\text {th }}$ impact are determined by using the concept of coefficient of restitution and conservation of momentum. These give:

$\dot{x}_{i a}=\left(\frac{1-\mu e}{1+\mu}\right) \dot{x}_{i b}+\mu\left(\frac{1+e}{1+\mu}\right) \dot{z}_{i b}$

$\dot{y}_{i a}=\left(\frac{1+e}{1+\mu}\right) \dot{x}_{i b}+\left(\frac{\mu-e}{1+\mu}\right) \dot{z}_{i b}$

Where $\mu$ is the mass ratio, $\mathrm{m} / \mathrm{M}$, and e is the coefficient of restitution. When contact occurs, the vibratory system can be shown using a two degree of freedom system. This system is shown in figure 2 , schematically.

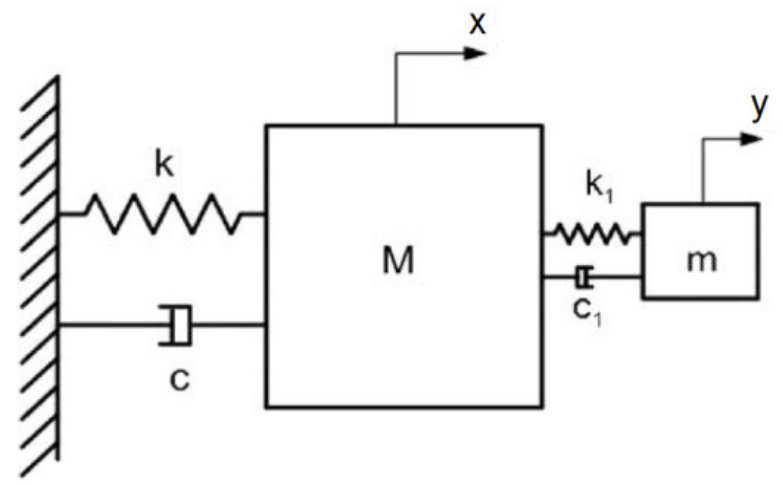

Figure2. Schematic while the impact mass colliding the primary mass.

Therefore, the governing equations of motion of the vibratory system should be written by two coupled equations which are shown in the following equations. 
$M \frac{d^{2} x}{d t^{2}}+k x+a(x-y)^{3}+b(x-y)=0$

$m \frac{d^{2} x}{d t^{2}}+k x+a(x-y)^{3}+b(x-y)=0$

In the above equations, the damping of system is considered negligible. It is reasonable because the contact duration with respect to the oscillation period of the primary mass is negligible. Nonlinear normal modes method (NNM) is used for solving the above displacement coupled equations. Consider $x=A \cos \omega t$ and $y=B \cos \omega t$ and substituting them into equations (8) and (9).Vanishing the resonant term results in:

$\frac{B}{A}=\frac{k-M \omega^{2}}{m \omega^{2}}$

(10)

Therefore equation (8) changes to:

$M \ddot{x}+k x+(1-C)^{3} x^{3}+b(1-C) x=0$

The above equation is a Doffing form of nonlinear equations, which can solve using iteration perturbation. This equation can be re-written as follows:

$\ddot{x}_{n+1}+\left(\frac{K+b-b C}{M}\right) x_{n+1}+\epsilon x_{n+1}\left(\frac{a(1-C)^{3}}{M}\right) x_{n}^{2}=0$

While $\epsilon$ is an artificial parameter, which is originally is equal to one. Consider:

$x=x_{0}+\epsilon x_{1}+\cdots$

Assume $x_{0}=\mathrm{X} \sin \omega \mathrm{t}$ as the first iteration. Therefore the extended form of angular frequency can be written in the following form:

$\frac{K+b-b C}{M}+\epsilon \frac{a X^{2}(1-C)^{3}}{2 M}=\omega^{2}+\epsilon C_{1}+\cdots$

Consider sentences which have same order of $\epsilon$. So the result for $\mathrm{x} 1$ can be written as follows:

$\ddot{x}_{1}+\omega^{2} x_{1}+C_{1} x_{0}-\frac{a X^{2}(1-C)^{3}}{2 M} x_{0} \cos 2 \omega t=0$

Substituting the first iteration into the above equation and vanishing the resonant term.

$\omega^{2}=\frac{1}{M}\left(K+b(1-C)+a\left(\frac{3}{4} X^{2}(1-C)^{3}\right)\right)$

Substituting the above equation into equation (10) results in:

$-\left(\frac{3}{4} X^{2} a \mu\right) D^{4}+\left(\frac{3}{4} X^{2} a(\mu-1) D^{3}-(b \mu) D^{2}+((b-K) \mu-b) D+K \mu=0\right.$

Where

$1-C=D$ and $\mu=\frac{m}{M}$ 
So, the coefficient $\mathrm{C}$ can be achieved by employing equation (18) and the period of the vibration can be obtained using equation (16) .Contact duration is equal to half of the period of vibration of impact mass when it collides with the barriers. Therefore the contact duration is equal to:

$t_{a}=\frac{2 \pi}{\sqrt{\frac{1}{M}\left(K+b(1-C)+a\left(\frac{3}{4} X^{2}\left((1-C)^{3}\right)\right)\right.}}$

And motion of primary mass between contacts can be formulated as follows:

$x\left(t^{\prime}\right)=e^{-\xi \omega t^{\prime}}\left[\frac{\dot{x}_{i a}+\xi\left(x_{i b}+V t_{a}\right)}{\sqrt{1-\xi^{2}}} \sin \left(\sqrt{1-\xi^{2}} \omega t^{\prime}\right)+\left(x_{i b}+V t_{a}\right) \cos \left(\sqrt{1-\xi^{2}} \omega t^{\prime}\right)\right.$

Where $V$ is the velocity of the primary mass when it collides with the impact mass. it is equal to:

$V=\frac{m}{m+M} \dot{y}_{i b}+\frac{m}{m+M} \dot{x}_{i b}$

A computer program in MATLAB was written to simulate equations (2) and (20) in real time. Consider the below parameters for the vibratory system with an impact damper.

Table1. Parameters for the vibratory system with an impact damper

\begin{tabular}{|c|c|}
\hline \multirow{2}{*}{ Masses } & $\mathrm{M}=2 \mathrm{~kg}$ \\
\cline { 2 - 2 } & $\mathrm{m}=0.1 \mathrm{~kg}$ \\
\hline Damping & $\mathrm{C}=0.01 \frac{\mathrm{N.s}}{\mathrm{m}}$ \\
\hline Stiffness & $\mathrm{K}=3000 \frac{\mathrm{N}}{\mathrm{m}}$ \\
\hline $\begin{array}{c}\text { Coefficient of } \\
\text { restitution }\end{array}$ & $\mathrm{e}=0.4$ \\
\hline
\end{tabular}

Figure 3 shows the displacement response of the main mass corresponding to the clearance $\mathrm{d}=0.01 \mathrm{~m}$ when it is subjected to the initial displacement $\mathrm{x}_{0}=\mathrm{y}_{0}=-0.007 \mathrm{~m}$. In this figure great effect of using impact damper for suppressing undesired vibration is clear.

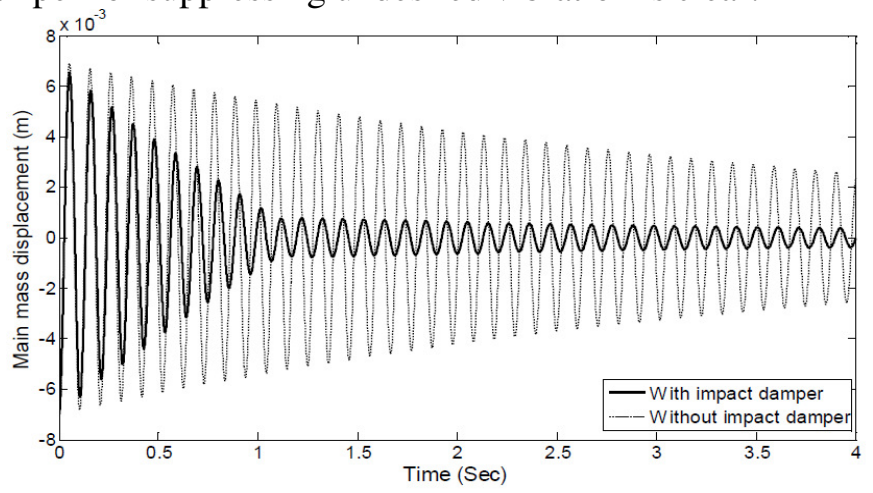

Figure3. Displacement response of the main mass with impact damper and without impact damper

Figure 3 shows significant effect of using the impact damper for suppressing undesired vibration of the main mass in free vibration but what is the effect of this system on forced vibration. 


\section{APPLICATION OF AN IMPACT DAMPER ON A 3R ROBOT:}

To begin this section, we pose the question: what is the effect of using an impact damper on vibration of a robot arm. For finding the answer of this question, a 3R robot is modeled using SimMechanics toolbox of MATLAB. The robot is equipped with a simple model of a single unit impact damper which is constructed using spring, mass and viscous damper. Two degrees of freedom of the robot are allocated to position control and another one is used for force controlling. Both of force and position are controlled using PID controllers and these separate controllers are synchronized using a hybrid control subsystem. Figure 3 shows the presented robot, schematically.

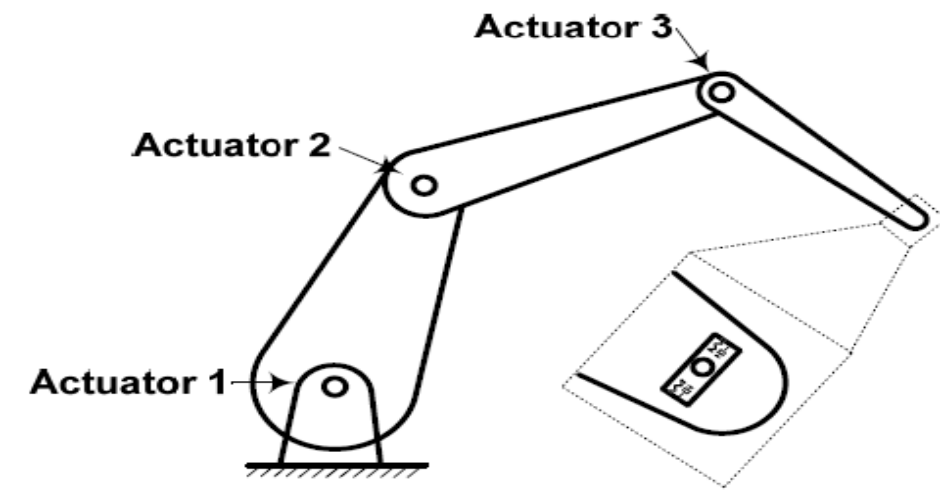

Figure4. Schematic of the $3 R$ robot

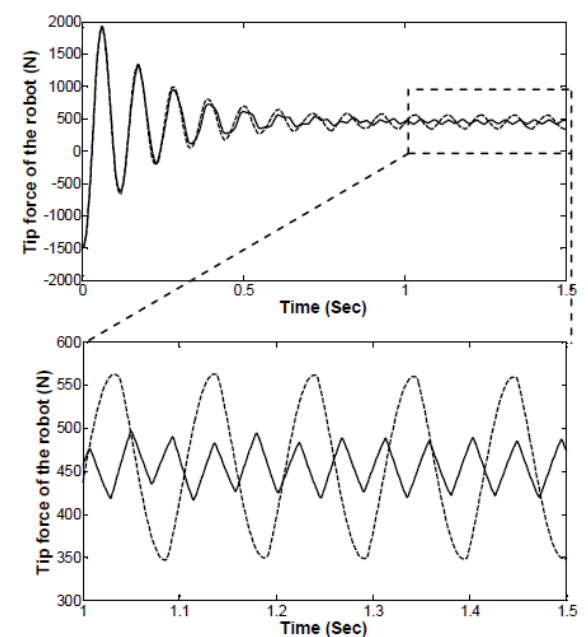

(a)

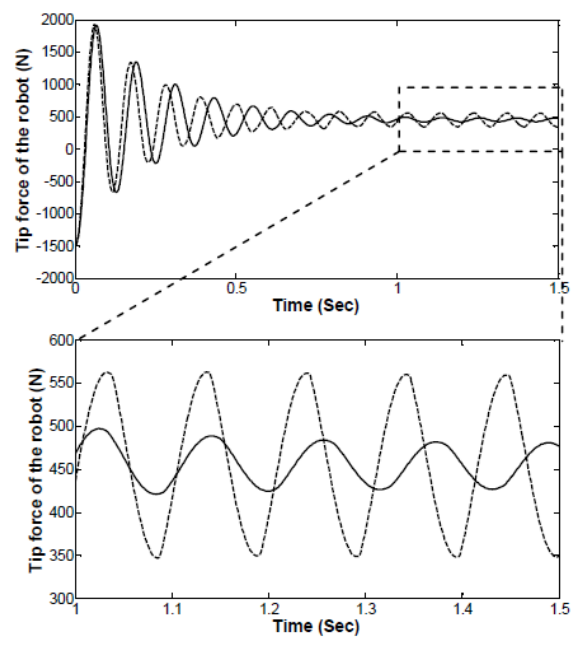

(b)

Figure4. Force response of the robot tip corresponding to clearance $\mathrm{d}=0.04 \mathrm{~m}$ and (a) $\mathrm{m}=0.4 \mathrm{Kg}$, (b) $\mathrm{m}=0.1 \mathrm{~kg} ;{ }_{-}$, with impact damper and ---, without impact damper

Figure 5 shows effectiveness of impact damper in case of forced vibration. In this figure, it is obvious that using the simple presented impact damper can attenuate the undesired vibration of robot tip and causes it works more accurate. 
International Journal of Recent advances in Mechanical Engineering (IJMECH) Vol.3, No.4, November 2014

\section{CONCLUSION}

This paper presents new analytical results for applying the impact damper to reduce structure vibration. A simple model of impact damper is constructed using spring, mass and viscous damper. The important feature to be carried out in the analysis of this model is that the deformation of an impact damper during the collision with the main mass can be formulated; therefore, the contact time is taken into consideration. This feature is important for a resilient rather than a rigid impact damper. The results clearly show that the impact damper can reduce the undesired vibration of primary mass in free vibration. Finally, the effect of impact damper for suppressing vibration of a robot tip is investigated. Using impact damper causes the robot works better than before. So, it is concluded that single unit impact dampers can suppress undesired vibration of forced vibratory systems.

\section{REFERENCES}

[1] Bapat, C.N. and S. Sankar, Single unit impact damper in free and forced vibration. Journal of Sound and Vibration, 1985. 99(1): p. 85-94.

[2] Cheng, C.C. and J.Y. Wang, Free vibration analysis of a resilient impact damper. International Journal of Mechanical Sciences, 2003. 45(4): p. 589-604.

[3] Cheng, J. and H. Xu, Inner mass impact damper for attenuating structure vibration. International Journal of Solids and Structures, 2006. 43(17): p.5355-5369.

[4] He, J.H., Iteration perturbation method for strongly nonlinear oscillations. Vibration Control, 2001. 7(5): p. 631.

[5] He, J.H., Some Asymptotic Methods for Strongly Nonlinear Equations. International Journal of Modern Physics B, 2006. 20(10): p. 1141-1199.

[6] J.E.Jam and A. A.Fard, Investigating Vibratory Behavior of Nonlinear Single Unit Impact Dampers Using Perturbation Method and Genetic Algorithm. International Journal of Aerospace Sciences, 2012. 1(2): p. 4.

[7] Zinjade, P.B. and A.K. Mallik, Impact damper for controlling friction-driven oscillations. Journal of Sound and Vibration, 2007. 306(1-2): p. 238-251.

[8] Duncan, M.R., C.R. Wassgren, and C.M. Krousgrill, The damping performance of a single particle impact damper. Journal of Sound and Vibration, 2005. 286(1-2): p. 123-144.

[9] Blazejczyk-Okolewska, B., Analysis of an impact damper of vibrations. Chaos, Solitons \& Fractals, 2001. 12(11): p. 1983-1988.

[10] Oledzki, A.A., Siwicki, I., Wisniewski, J., Impact dampers in application for tube, rod and rope structures. Mechanism \& Mach. Theory, 1999. 34(2): p. 243-253.

[11] Ema, S., Marui, E., Damping characteristics of an impact damper and its application. Int. J. Mach. Tools \& Manuf., 1996. 36(3): p. 293-306.

[12] Son, L., et al., Experiment of Shock Vibration Control Using Active Momentum Exchange Impact Damper. Journal of Vibration and Control, 2010. 16(1): p. 49-64. 